\title{
Side Information Refinement for Transform domain Distributed Video Coding
}

\author{
Sangwoo Lee and Sang Ju Park ${ }^{1}$ \\ Department of Electronics, Information and Communication Engineering, \\ Hongik University, 72-1, Sangsu-Dong, Mapo-Gu, Seoul 121-791, \\ Republic of Korea \\ klassesw@mail.hongik.ac.kr,sjpark@hongik.ac.kr
}

\begin{abstract}
A new side information refinement method for transform domain distributed video coding $(D V C)$ is proposed. In DVC, side information is the frame constructed from key frames which are intra coded. It is utilized as the initial estimate of source symbols of Wyner-Ziv coded frame which is not transmitted to the decoder. Errors in the estimated frames are to be corrected by the decoder using the parity bits which are the only information received from the encoder. Quality of decoder generated side information is one of the most important factors deciding the overall performance of the decoder. A new refinement method of improving the quality of side information of the DVC decoder is proposed. The side information frame is refined at the end of each transform band decoding stage followed by a conventional bi-directional motion estimation and compensation technique. Proposed side information refinement technique consequently improves the quality of final reconstructed pictures.
\end{abstract}

Keywords: Video compression, Distributed video coding, DVC, Side information

\section{Introduction}

In previously released video compression techniques, most of the required arithmetic operations for information compression are performed at the encoder. These computation intensive encoding techniques are developed for DVD, Blu-Ray and the public TV broadcasting service. These encoder side computation intensive video coding techniques, however, are not well suited for small multimedia devices because of limited resources, such as the insufficient computing power of a battery powered device.

Distributed video coding (DVC) is a video coding paradigm and a promising candidate for overcoming such limitations. Distributed lossless source coding techniques began with the Slepian-Wolf theorem in the 1970s [1]. It was then extended to lossy compression along with quantization after the study of Wyner and Ziv [2]. The key result of these works is that statistically dependent signals can be separately encoded and jointly decoded at the same rate used in a system where joint encoding is utilized. DVC's based on these pioneering works, have been studied by many researchers including Stanford University [3], Berkeley

${ }^{1}$ Corresponding Author 
University [4] and Europe's joint research group [5, 6]. For more complete DVC related works please refer to the review papers $[7,8,9]$.

In the DVC encoder there is no loop architecture, which is an essential and complex part of any hybrid video coding techniques such as MPEG2 and H.264/AVC. In such conventional hybrid video coding techniques, motion estimation and compensation are the key components of the encoder. Motion estimation is very computation intensive process and takes up most of the encoding time of the conventional hybrid video encoder. Estimated motion information, namely motion vectors are transmitted to the decoder and decoder performs only motion compensation. On the other hand, in DVC structure, computation intensive motion estimation is performed at the decoder. Therefore, DVC encoder structure is simple and well suited for encoders with limited resources such as mobile devices.

Figure 1 shows the block diagram of general DVC codec.

At the encoder, input frames are divided into two types: key frames and Wyner-Ziv (WZ) frames. The key frames are encoded with a conventional intra frame encoder such as H.264/AVC intra mode. WZ frames are also coded independently of the key frames with a distributed coder, which is a simple error correction encoder such as Turbo coder or Low Density Parity Check (LDPC) coder. In transform domain DVC, each WZ frame is first transformed block by block by a conventional spatial domain block transform matrix such as discrete cosine transform.

The block transform coefficients are then quantized and zig-zag scanned and the quantized coefficients located at the same locations in the transform bands of the entire frame are collected in raster scan order to form the coefficients band plane. This process is called the band plane extraction procedure and is shown in Figure 2. In Figure 2, the numbers shown in the left blocks are orders of zig-zag scan. $B_{1}$ to $B_{16}$ are extracted band planes. Each band plane is encoded using a systematic error correction encoder such as Turbo code or LDPC code. Unlike most other error correction code applications, only the parity bits are transmitted to the decoder. This is how compression is achieved in DVC structure. Source bits are not transmitted. It is decoder's job to find estimate of source bits using reconstructed key frame data.

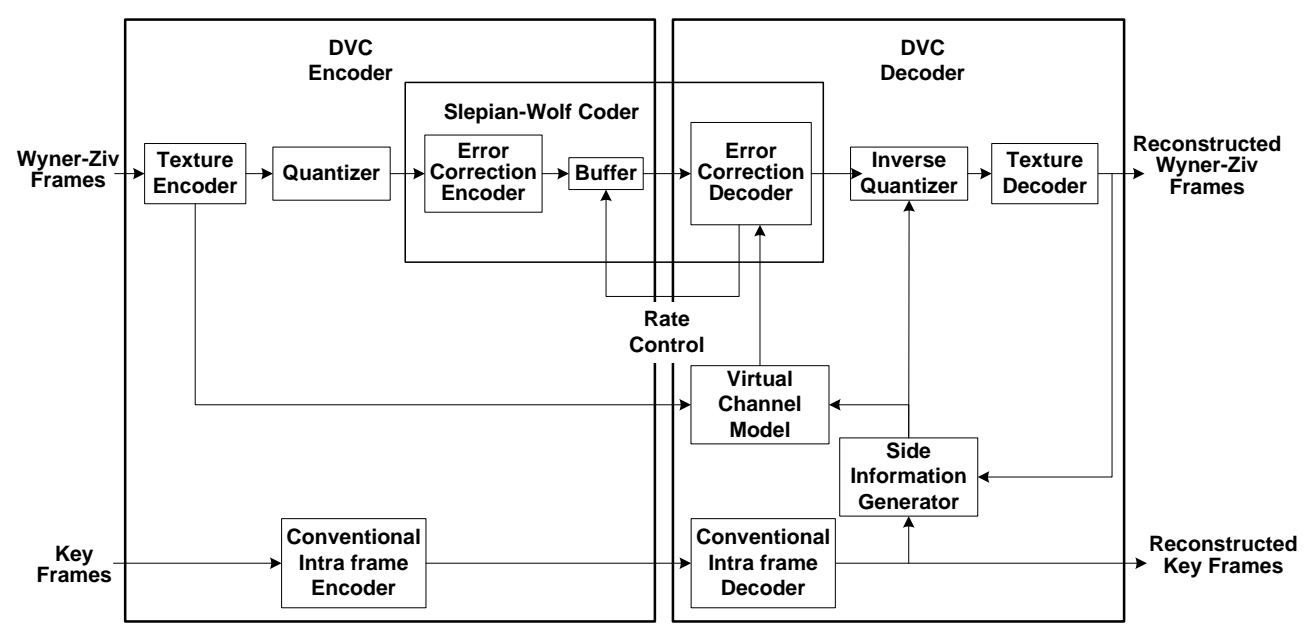

Figure 1. The general block diagram of a DVC codec 


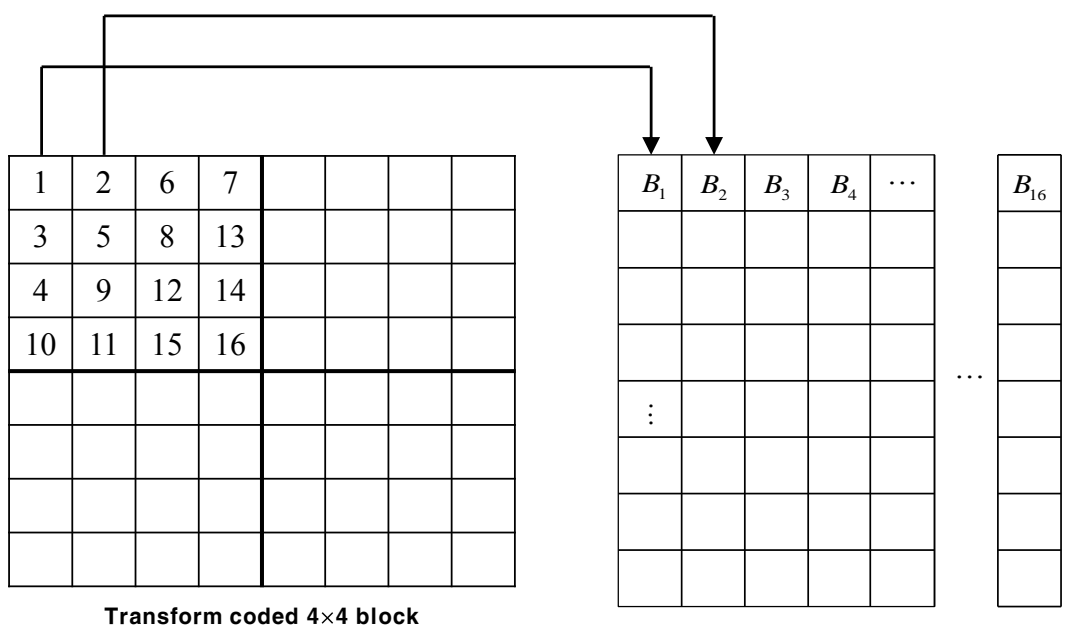

Figure 2. Band plane extraction and zig-zag scan procedure

In other words, WZ encoding is relatively simple operations of error correction encoding, while the DVC decoder has to carry out most of the complex operations to jointly decode the separately encoded frames, including error correction decoding and motion estimation and compensation namely side information generation, which were components of the encoder in other conventional hybrid video coding methods.

The most important components of the DVC decoder are side information generator and error correcting decoder. As mentioned earlier, side information generation at the decoder is the process of finding approximations of source symbols not transmitted from the encoder. It is similar to the conventional encoder side motion estimation and compensation. It is, however, more difficult than the conventional method, since no reference picture data are available for the decoder. Rather, it is similar to the process of frame rate up conversion. Quality of generated side information is the key element of deciding the overall DVC performance.

In [10], we proposed a side information update method which is as follows.

The error correction decoder starts decoding each transform coefficient band by bit plane level. The most significant bit plane (MSB) is decoded first and the least significant bit plane (LSB) is decoded lastly. We can improve the accuracy of side information by updating side information, after decoding each bit plane.

For example the quantization index of 2057 ("WZ" in Figure 3(a)) is 8 with 16 level (4-bit) uniform quantizer. The binary representation of quantization index 8 is " 1000 " " $\mathrm{QI}$ : 8" in Figure 3(a)). Suppose that the corresponding side information value is 1849 (" SI" in Figure 3(a)), then binary representation of 1849 is "0111 00111001 " and the quantization index of same 16 level quantizer of that side information value is 7 ("QI: 7" in Figure 3(a)). The first bit of decoded quantization index changes from " 0 " to " 1 " if error correction decoder corrects the error. Since the decoded bit is different from corresponding bit of side information, it is not reasonable to use the same side information value when decoding the next bit plane. In this case, it is appropriate that the side information is updated using previously decoded bit for decoding the subsequent bit planes. 
The updated value of side information is decided by both the values of quantization index and its quantization interval. Suppose for example that the quantization index of side information is q and its certain bit is corrected, i.e., changed from " 0 " to " 1 " . Then the upper limit of the quantization interval of quantization index q-1 becomes the updated side information value. On the other way around, if a bit is corrected from " 1 " to " 0 ", the updated side information value is the lower limit of the quantization interval corresponding to the quantization index $\mathrm{q}+1$.

In above numerical example, a bit is corrected from " 0 " to " 1 ". So the updated side information value is 2048 which is lower limit of quantization interval corresponding to quantization index 8. This process is illustrated Figure 3(a).

As another example, if the transform coefficient is 1849("WZ" in Figure 3(b)) then its quantization index is 7(“QI: 7" in Figure 3(b)). If the side information value is 2057("SI" in Figure 3(b)), then its quantization index is $8($ "QI: 8 " in Figure 3(b)). If the first bit is corrected from " 1 " to " 0 ", the side information is updated to 2047 which is upper bound of the 7-th quantization interval as depicted in Figure 3(b). When a bit is not changed after error correction decoding, original side information value is kept. The remaining bit planes are decoded without modifying the value.

In this paper, we propose a new refinement method to improve the quality of side information generated at a DVC decoder. The proposed side information refinement is to improve the quality of the side information at the band-plane level.

This paper is organized as follows. In Section 2, we outline the proposed method. In Section 3, we experimentally verify the validity of the proposed method. Finally in Section 4, we provide conclusions.

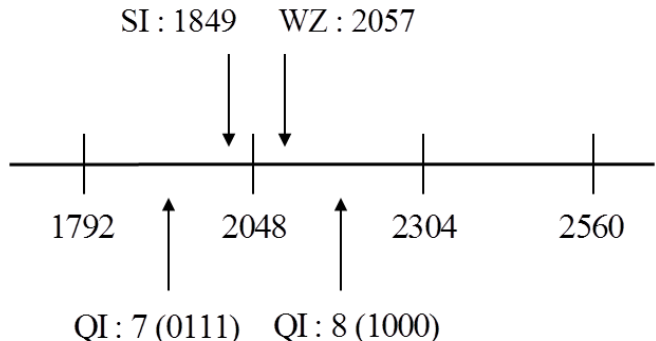

QI : 7 (0111) QI : 8(1000)

SI : 1849 (01110011 1001)

SI : $2048(100000000000)$

(a)

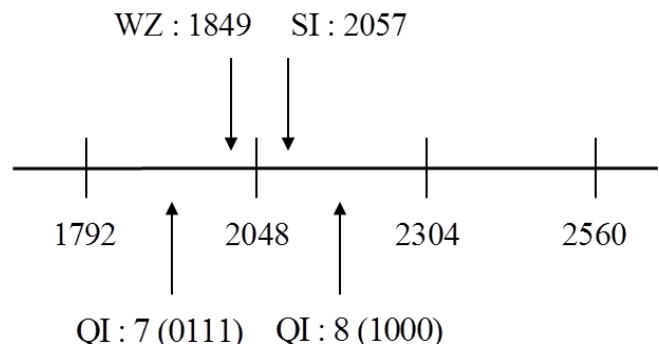

SI : 2057 (1000 0000 1001)

SI : 2047 (0111 11111111$)$

(b)

Figure 3. Examples of Side information update process 


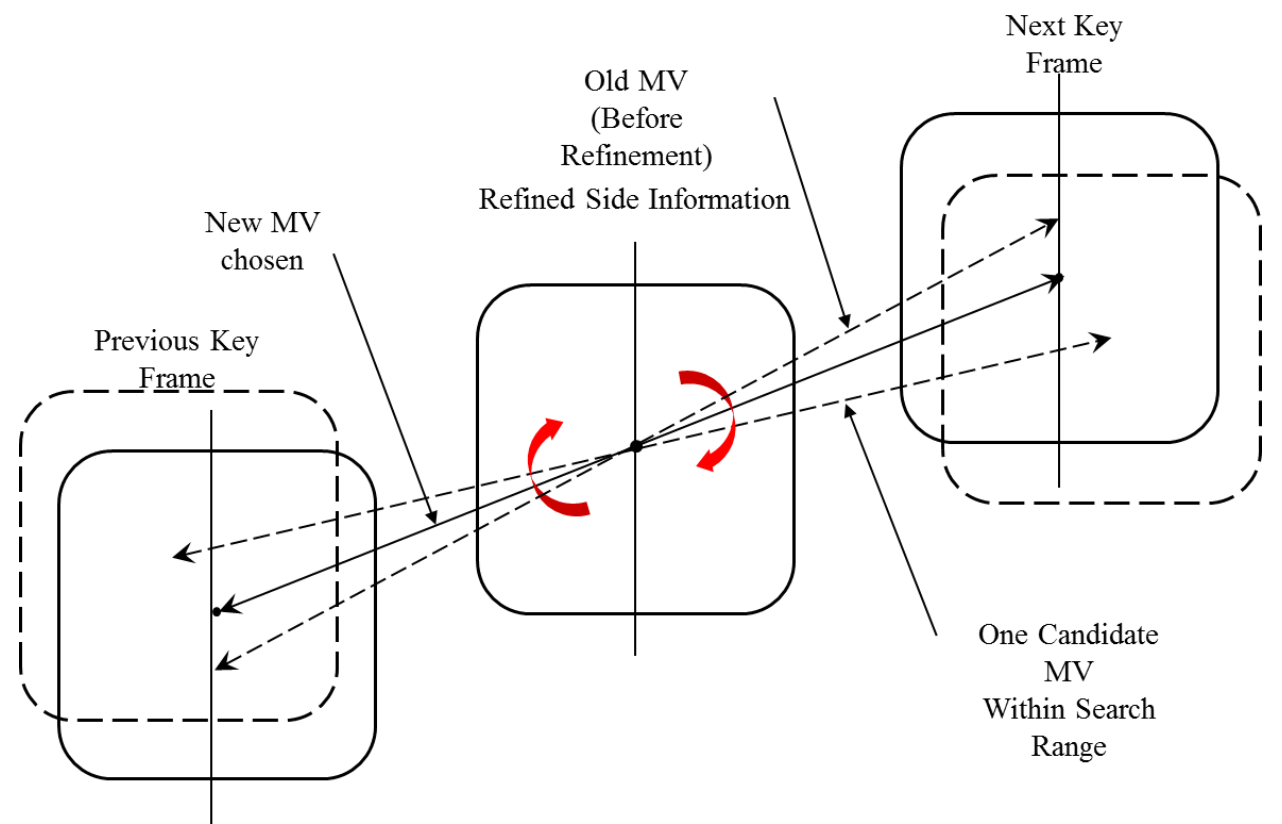

Figure 4. Side information refinement

\section{Side Information Refinement Method}

In the existing transform domain DVC codec, the side information, once generated, is used continuously until one frame decoding is completed on the error correcting decoder and the inverse quantizer.

In our proposed method as illustrated in Figure 4, the side information is refined after decoding each zig-zag scanned transform band, and it is subsequently utilized for the next transform band decoding.

The DVC's error correcting decoder corrects errors in each transform band of side information using the transmitted parity bits. Therefore, errors in decoded transform band should be less than that of the initial side information, which is simply the uncorrected estimate. Therefore, if motion estimation and compensation is performed again, newly generated motion compensated frame is expected to serve as better side information.

This refined transform domain side information is dequantized and inverse transformed to obtained refined pixel domain side information. It is then used to generate new, more refined side information, resulting in newly generated side information that has reduced distortion when compared to the previous side information. Moreover, new refined side information generation can be done using conventional bi-directional motion estimation and compensation technique. Better side information contributes to improving the quality of the decoded frame because it is reused at inverse quantizer (pixel value reconstruction process).

In addition, we can then reduce the amount of parity bits to be transmitted for the next band by reducing the errors in the side information. In other words, proposed side information refinement step not only improves the decoded picture quality but also it does so in a reduced rates. 
The 4 times magnified and cropped version of reconstructed frames with and without the proposed side information refinement are presented in Figure 5. Upper pictures are reconstructed WZ frames without side information refinement. Bottom pictures are reconstructed WZ frames after applying proposed side information refinement process.

It clearly shows the advantage of the side information refinement process. The distortions at bows of small boat in coastguard sequence and bag carried by a person in hall sequence are mitigated by the refinement steps. Numerical experimental results are given in Section 3.

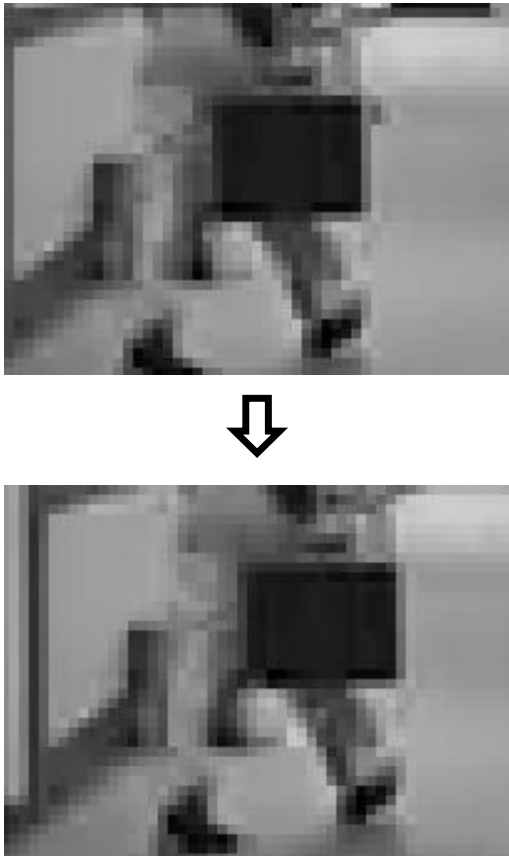

(a)
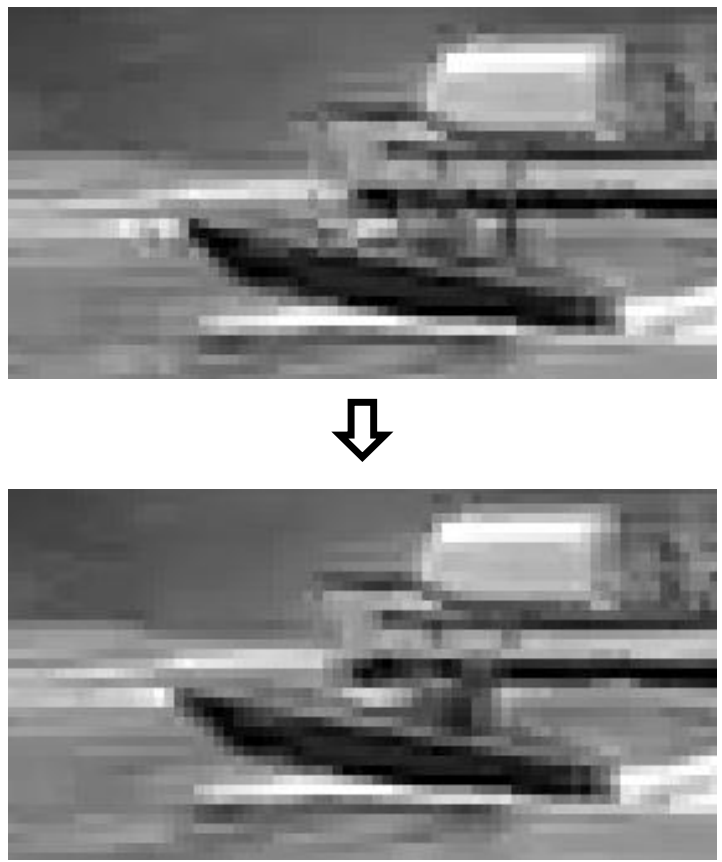

(b)

Figure 5. The reconstructed frames with and without side information refinement procedure. (a) Hall Sequence (b) Coastguard Sequence

\section{Experimental Results}

We implemented our DVC codec using MATLAB to test the performance of our methods. The test conditions are the same as the Discover codec $[5,6]$ and test sequences are "Coastguard" and "Hall". The rate and distortion performance results of the proposed methods are presented in table 1 and Figure 5 in previous section. Table 1 shows the changes of rate and quality (PSNR) respectively before and after applying the proposed side information refinement method. PSNR improvement ranges from $0.07 \mathrm{~dB}$ to $0.99 \mathrm{~dB}$ depending on the quantization matrix and sequences. Encoding rates are also decreased by from $0.01 \mathrm{Kbits} /$ frame to $0.94 \mathrm{kbits} /$ frame. Improvements of rate distortion factors are more noticeable as the quantizer step size decreases. 


\section{Table 1. Rate and distortion performance comparison}

(a) Coastguard

\begin{tabular}{c|ccc|ccc}
\hline \multirow{2}{*}{ Q } & \multicolumn{3}{|c|}{ PSNR $(\mathrm{dB})$} & \multicolumn{3}{c}{ Rate (Kbit/frame) } \\
\cline { 2 - 7 } Matrix & Previous & Proposed & $\Delta$ & Previous & Proposed & $\Delta$ \\
\hline Q1 & 27.47 & 27.74 & 0.27 & 3.62 & 3.59 & -0.03 \\
Q2 & 27.90 & 28.23 & 0.33 & 5.02 & 4.98 & -0.04 \\
Q3 & 27.90 & 28.25 & 0.35 & 5.85 & 5.79 & -0.06 \\
Q4 & 28.94 & 29.42 & 0.48 & 9.55 & 9.37 & -0.18 \\
Q5 & 29.13 & 29.69 & 0.56 & 10.45 & 10.25 & -0.20 \\
Q6 & 29.52 & 30.23 & 0.71 & 14.84 & 14.50 & -0.34 \\
Q7 & 29.99 & 30.75 & 0.76 & 20.06 & 19.53 & -0.53 \\
Q8 & 30.60 & 31.59 & 0.99 & 35.57 & 34.63 & -0.94 \\
\hline
\end{tabular}

(b) Hall

\begin{tabular}{c|ccc|ccc}
\hline \multirow{2}{*}{$\begin{array}{c}\text { Q } \\
\text { Matrix }\end{array}$} & \multicolumn{3}{|c|}{ PSNR (dB) } & \multicolumn{3}{c}{ Rate (Kbit/frame) } \\
\cline { 2 - 7 } & Previous & Proposed & $\Delta$ & Previous & Proposed & $\Delta$ \\
\hline Q1 & 31.22 & 31.28 & 0.07 & 2.69 & 2.68 & -0.01 \\
Q2 & 31.58 & 31.70 & 0.12 & 4.02 & 4.02 & -0.01 \\
Q3 & 31.60 & 31.71 & 0.11 & 4.98 & 4.98 & 0.00 \\
Q4 & 33.34 & 33.49 & 0.15 & 7.09 & 7.06 & -0.02 \\
Q5 & 33.35 & 33.50 & 0.15 & 8.45 & 8.43 & -0.03 \\
Q6 & 34.35 & 34.50 & 0.15 & 12.05 & 12.01 & -0.04 \\
Q7 & 35.19 & 35.42 & 0.23 & 14.17 & 14.10 & -0.07 \\
Q8 & 36.62 & 36.88 & 0.26 & 20.67 & 20.55 & -0.12 \\
\hline
\end{tabular}

Rate distortion performances are compared and presented in Figure 6. It shows the results of applying side information refinement method proposed in this paper and/or previously reported side information update method in [10]. The PSNR of final decoded sequences are improved by the side information refinement method. As a result, the RD curve moves toward the upper side. As previously noted, bit rate is decreased by applying the side information update method [10]. In other words, RD curves are move to the left of conventional method. It means that our methods can improve RD performance of DVC codec. 


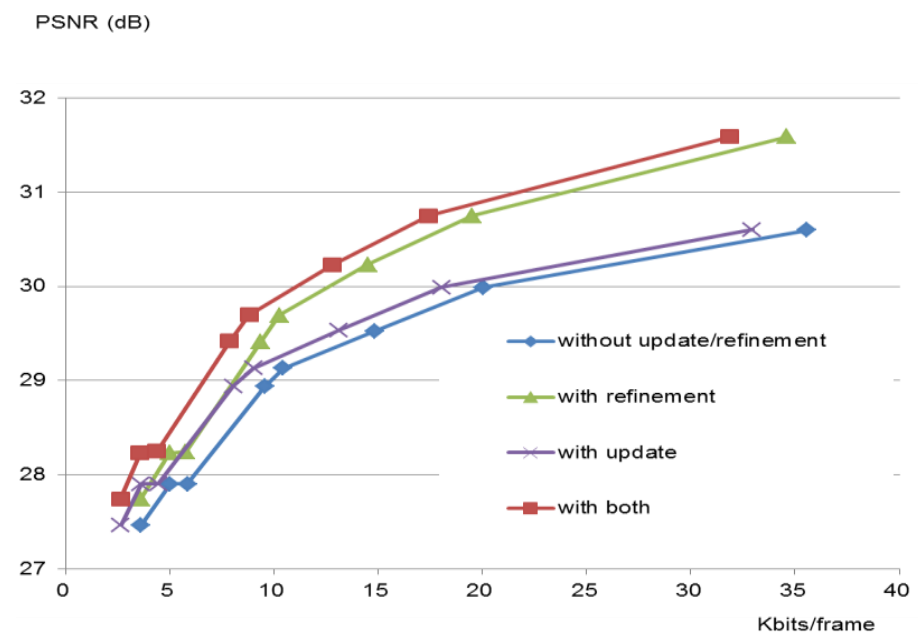

(a) Coastguard

PSNR (dB)

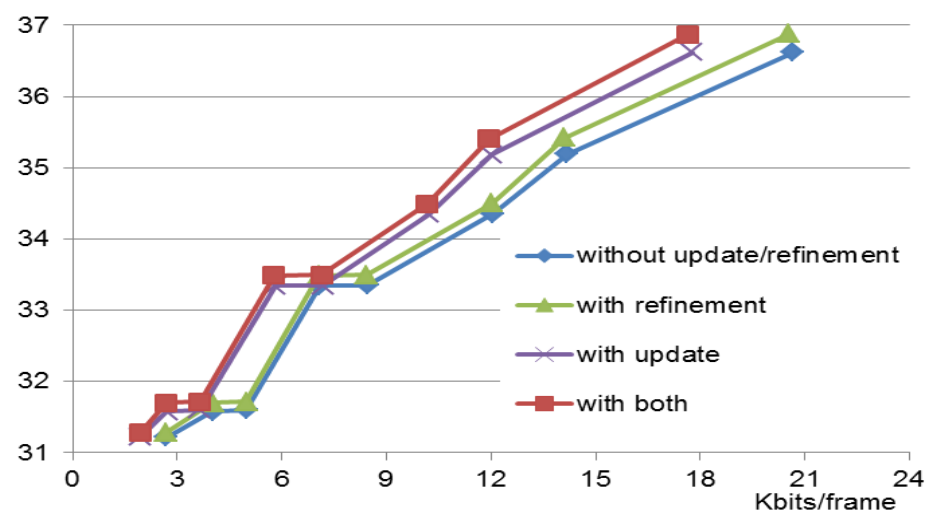

(b) Hall

Figure 6. Rate and distortion performance of proposed methods

\section{Conclusions}

In this paper we proposed a side information refinement method improving the performance of DVC codec. The PSNR of final decoded sequences is increased by side information refinement method. Furthermore the decoding complexity of error correcting decoder can be reduced using quality improved side information. We are expecting that overall DVC performance can be improved using proposed methods and previously proposed methods $[10,11]$.

\section{Acknowledgements}

This research was supported by Basic Science Research Program through the National Research Foundation of Korea (NRF) funded by the Ministry of Education, Science and Technology (20110025927). 


\section{References}

[1] J. D. Slepian and J. K. Wolf, "Noiseless coding of correlated information sources", IEEE Transactions on Information Theory, vol. 19, no. 4, (1973), pp. 471-480.

[2] A. D. Wyner and J. Ziv, "The rate-distortion function for source coding with side information at the decoder", IEEE Transactions on Information Theory, vol. 22, no. 1, (1976), pp. 1-10.

[3] B. Girod, A. M. Aaron, S. Rane and D. R-. Monedero, "Distributed Video Coding", Proceedings of the IEEE, vol. 93, no. 1, (2005), pp. 71-83.

[4] R. Puri and K. Ramchandram, "PRISM: An uplink-friendly multimedia coding paradigm", Proceedings of the International Conference on Acoustics, Speech and Signal Processing, Hong Kong, vol. 4, (2001), pp. IV859-9.

[5] http://www.discoverdvc.org.

[6] X. Artigas, J. Ascenso, M. Dalai, S. Klomp, D. Kubasov and M. Ouaret, "The DISCOVER codec: Architecture, Techniques and Evaluation”, Proceedings of the Picture Coding Symposium, Lisbon, Portugal, (2007).

[7] G. R. Esmaili and P. C. Cosman, "Wyner-Ziv Video Coding With Classified Correlation Noise Estimation and Key Frame Coding Mode Selection”, IEEE Transactions on Image Processing, vol. 20, no. 9, (2011), pp. 2463-2474.

[8] C. Guillemot, F. Pereira and L. Torres, "Editorial: Special issue on distributed video coding", Signal Processing: Image Communication, vol. 23, no. 5, (2008).

[9] P. L. Dragotti and M. Gastpar, "Distributed Source Coding: Theory, Algorithms and Applications", Academic Press, (2009).

[10] S. Lee and S. J. Park, "Side Information Update Method of Error Correction Decoder for Distributed Video Coding", International Journal of Multimedia and Ubiquitous Engineering, vol. 7, no. 2, (2012), pp. 171-176.

[11] H. Jang, S. Lee and S. J. Park, "On Using Redundant Parity Check Equation for LDPCA decoding in Distributed Video Coding", International Journal of Advanced Science and Technology, vol. 42, (2012), pp. 11-18.

[12] A. J. Santoso, L. E. Nugroho, G. B. Suparta and R. Hidayat, "Color Image Compression Using Orthogonal Wavelet Viewed From Decomposition Level and Peak Signal to Noise Ratio", International Journal of Advanced Science and Technology, vol. 31, (2011), pp. 81-91.

\section{Authors}

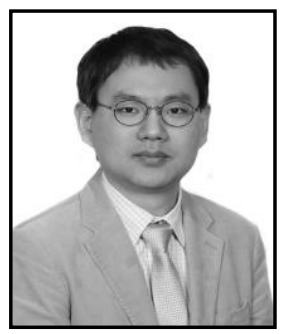

\section{Sangwoo Lee}

He received the B.S., M.S. and Ph.D. degrees in School of Electronic \& Electrical Engineering from Hongik University, Korea, in 2003, 2006 and 2012 respectively. His research interests are multimedia data processing and compression.

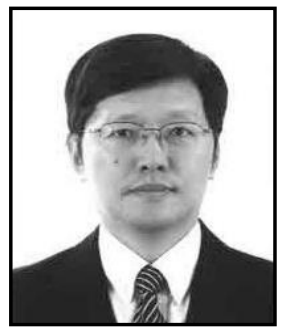

\section{Sang Ju Park}

He received the B.S. and M.S. degrees in electronics engineer-ing from Seoul National University, Korea, in 1984 and 1986 respectively and the Ph.D. degree in electrical engineering from Stanford University, Stanford, CA, in 1992. He was an MTS at PRPA(Philips Research Palo Alto) and Philips Semiconductors, Sunnyvale during 1992-1995. Since March 1996, he has been at Hongik University, where he is currently a professor. His research interests are multimedia data processing and compression. 
International Journal of Multimedia and Ubiquitous Engineering Vol.8, No.5 (2013) 